\title{
Expression of cytokeratin 7 predicts survival in stage I/IIA/IIB squamous cell carcinoma of the esophagus
}

\author{
ATSUSHI YAMADA ${ }^{1,4}$, HIROKI SASAKI ${ }^{3}$, KAZUHIKO AOYAGI $^{3}$, MASAYUKI SANO $^{3}$, \\ SATOSHI FUJII ${ }^{1}$, HIROYUKI DAIKO ${ }^{2}$, MITSUYO NISHIMURA ${ }^{2}$, TERUHIKO YOSHIDA ${ }^{3}$, \\ TSUTOMU CHIBA ${ }^{4}$ and ATSUSHI OCHIAI ${ }^{1}$
}

\begin{abstract}
${ }^{1}$ Pathology Division, Research Center for Innovative Oncology, ${ }^{2}$ Department of Surgery, National Cancer Center Hospital East, 6-3-1 Kashiwanoha, Kashiwa, Chiba 277-8577; ${ }^{3}$ Genetics Division, Research Institute, National Cancer Center, 5-1-1 Tsukiji, Chuou-ku, Tokyo 104-0045; ${ }^{4}$ Department of Gastroenterology and Hepatology, Graduate School of Medicine, Kyoto University, 54 Shogoin-kawara-cho, Sakyou-ku, Kyoto 606-8507, Japan
\end{abstract}

Received June 20, 2008; Accepted August 1, 2008

DOI: 10.3892/or_00000104

\begin{abstract}
Squamous cell carcinoma of the esophagus (ESCC) is one of the most aggressive carcinomas, and it occasionally recurs even in the early stages. Identifying biomarkers that enable stratification of patient survival, especially in the early stages, is important to establish better treatment protocols. By performing microarray analyses, we recently discovered that expression of cytokeratin (CK) 7 and CK14 is related to the clinical outcome of ESCC patients. The aim of the present study was to evaluate the significance of CK7 and CK14 expression in a series of ESCC cases. Tissue sections from 126 surgically resected ESCCs were immunostained for CK7 and CK14, and their expression was evaluated in relation to the clinicopathological findings and outcome. Expression of CK7 and CK14 was detected in 28 (22\%) and 106 (84\%), respectively, of the 126 ESCCs. CK7-positive was associated with poor differentiation $(\mathrm{p}=0.049)$, and CK14negative was associated with poor differentiation $(\mathrm{p}<0.001)$, lymphatic invasion $(\mathrm{p}=0.027)$ and advanced stages $(\mathrm{p}=0.043)$ of ESCCs. CK7-positive and CK14-negative associated a poor outcome ( $\mathrm{p}=0.014$ and 0.013 , respectively). Among the stage I/IIA/IIB patients, CK7 expression was a significant predictor of worse survival rate $(\mathrm{p}<0.001)$. The 5 -year overall survival rate of stage I/IIA/IIB patients with CK7-positive and CK7-negative tumors was 50.0 and $90.3 \%$, respectively. Univariate and multivariate analyses of the stage I/IIA/IIB
\end{abstract}

Correspondence to: Dr Atsushi Ochiai, Pathology Division, Research Center for Innovative Oncology, National Cancer Center Hospital East, 6-3-1 Kashiwanoha, Kashiwa, Chiba 277-8577, Japan

E-mail: aochiai@east.ncc.go.jp

Key words: squamous cell carcinoma of the esophagus, cytokeratin 7 , cytokeratin 14 , immunohistochemistry, survival, early stage
ESCC patients by the Cox proportional hazards model showed that lymphatic invasion and CK7 expression were significant prognostic factors. Thus, CK7 expression was a useful biomarker for predicting the outcome of stage I/IIA/IIB ESCC.

\section{Introduction}

Squamous cell carcinoma of the esophagus (ESCC) is one of the most aggressive carcinomas and generally has an unfavorable prognosis. Despite recent advances in diagnosis and surgery, ESCC remains a major cause of cancer mortality $(1,2)$. Multimodal treatment strategies that combine chemotherapy and/or radiotherapy with surgery have been assessed to improve survival $(2,3)$, and some authors have demonstrated efficacy of the combination therapy in ESCC patients $(4,5)$. However, the ESCC patients who benefit most by such multimodal strategies have yet to be identified. Stratifying patients according to clinical outcome is essential to devising treatment tailored to individual patients. Although the TNM staging system is the internationally accepted method for determining the prognosis of esophageal cancer, ESCC is marked by occasional recurrence even in the early stages of the disease. Nozoe et al reported recurrence in 10 of 83 stage I ESCC patients after surgical resection (6), and 5 -year survival rates after surgical resection in stage I, IIA and IIB of 88.0, 44.2 and $42.8 \%$, respectively (1). Thus, the early TNM stage ESCC cases contain a subgroup of cases with poor clinical outcome. It is important to establish a better treatment protocol for early stage patients, because if intensive multimodal treatment is performed in appropriately selected patients, it may improve their survival. Stratifying early stage ESCC patients according to clinical outcome is necessary to be able to choose proper subjects for intensive treatment, but the TNM system seems inadequate for this purpose. Even some stage I ESCCs recur, indicating the existence of factors that affect prognosis independent of the TNM system. Thus, it is crucial to identify biomarkers for the clinical outcome of ESCC, in addition to using the TNM staging system. 
By performing mircoarray analyses, we recently discovered that expression of cytokeratin (CK) 7 and CK14 is involved in the clinical outcome of ESCC patients (Sano et al, unpublished data). CKs are filament-forming proteins in epithelial cells and are essential to normal tissue structure and function (7). Different subsets of CKs are expressed depending on the epithelial cell type and the degree of differentiation of the cells. Since the CK profile is usually preserved during neoplastic transformation, its expression has been widely used to fingerprint various carcinomas $(8,9)$. CK7 is expressed in normal ductal epithelia, including the epithelium of the glandular ducts of the esophagus, but not in the esophageal mucosa. In neoplastic tissue, CK7 expression is primarily observed in primary adenocarcinomas of many organs, but it is also expressed in a subset of ESCCs $(8,10)$. CK14 is expressed in the basal cells of normal stratified squamous epithelia and the vast majority of squamous cell carcinomas, including $\operatorname{ESCC}(8,11,12)$. Several authors demonstrated an association between CK7 expression in tumors and a poor outcome, including in patients with adenocarcinoma of the lung (13) and hepatocellular carcinoma (14). However, the clinical implications of CK7 and CK14 expression in ESCCs have not been investigated. The aim of this study was to elucidate the clinical significance of expression of CK7 and CK14 detected by immunohistochemistry in relation to the clinicopathological findings and outcome of ESCC patients, especially in the relatively early stages of the disease.

\section{Materials and methods}

Patients and follow-up. A total of 126 consecutive ESCC patients who underwent surgical resection with curative intent between January 1992 and December 2003 at the National Cancer Center Hospital East were enrolled in this study. Patients with simultaneous multiple esophageal cancers, patients with uncontrolled malignant tumors in other organs, patients who received any adjuvant therapy prior to surgery, and patients who died within 30 days after surgery were excluded from this study. This study was approved by the Institutional Review Board at the National Cancer Center.

There were 107 men and 19 women, and their median age was 62 years (range, 40-78). Clinicopathological findings were assessed based on the International Union Against Cancer (UICC) TNM staging system (15). There were 31 stage I cases, 16 stage IIA cases, 31 stage IIB cases, 43 stage III cases and 5 stage IV cases. Histologic grade according to the criteria of the World Health Organization (16) was well differentiated in $9(7 \%)$ cases, moderately differentiated in $94(75 \%)$ and poorly differentiated in $23(18 \%)$.

The median follow-up period of the surviving patients was 70 months (range, 2-158). The median overall survival time and median disease-free survival time were 57 months (range, 1-158) and 51 months (range, 1-158), respectively. The 5-year overall survival rate of the stage I/IIA/IIB group, stage III/IV group and entire group as a whole was 78.7, 40.3 and $64.4 \%$, respectively.

Immunohistochemistry. All resected specimens were fixed in either $10 \%$ formalin or methanol, and embedded in paraffin.
Sections $4-\mu \mathrm{m}$ thick were cut from paraffin embedded block that contained the most representative area of the tumor and used for immunohistochemical staining. The sections were deparaffinized in xylene, dehydrated in a graded series of ethanol, and immersed for $15 \mathrm{~min}$ in methanol containing $0.3 \%$ hydrogen peroxide to inhibit endogenous peroxidase activity. The slides were heated in citrate buffer $(\mathrm{pH} \mathrm{6.0)}$ at $95^{\circ} \mathrm{C}$ for $20 \mathrm{~min}$ in a microwave oven for antigen retrieval, and then allowed to cool to room temperature. Non-specific binding was blocked by preincubation with $2 \%$ normal swine serum in phosphate-buffered saline (PBS) for $60 \mathrm{~min}$ at room temperature. After incubating the slides with the respective primary antibodies overnight at $4^{\circ} \mathrm{C}$, the slides were washed three times with PBS and incubated with EnVision (Dako) for $1 \mathrm{~h}$ at room temperature. The sections were then visualized with 3,3'-diaminobenzidine tetrahydrochloride in $50 \mathrm{mM}$ Tris-buffer ( $\mathrm{pH} 7.6)$ containing $0.3 \%$ hydrogen peroxide as the chromogen, and counterstained with hematoxylin. The antibodies used in this study were anti-CK7 (mouse monoclonal, clone OV-TL, Dako, 1:50) and anti-CK14 (mouse monoclonal, clone LL002, Novocastra, 1:20). Normal esophageal gland duct cells and normal esophageal epithelial basal cells served as an internal positive control for CK7 and CK14, respectively, to validate the immunohistochemical staining in each case. Negative controls were prepared by omitting the primary antibodies.

The proportion of tumor cells that stained immunohistochemically positive in each section was determined semi-quantitatively. When $\geq 10 \%$ of the cells stained positive, the tumor was considered positive for expression of the antigen, and if $<10 \%$ of the cells stained positive, the tumor was considered negative (9).

Statistical analysis. Associations between expression of CK7 and CK14 and the clinicopathological features were evaluated using the $\chi^{2}$ test and Fisher's exact test. Cumulative survival curves were drawn by the Kaplan-Meier method, and differences between curves were analyzed by the log-rank test. The Cox proportional hazards model was used for univariate and multivariate analyses of prognostic factors. All tests were two-sided and a p-value of $<0.05$ was considered significant. All analyses were carried out using Statview version 5.0 for Windows software (SAS Institute Inc., Cary, NC, USA).

\section{Results}

CK7 expression and clinicopathological parameters of ESCC. CK7 expression was observed in the cytoplasm of non-neoplastic duct and glandular epithelium of esophageal glands (Fig. 1A) and in the cytoplasm of cancer cells in a subset of ESCCs. Some cancer cells showed strong CK7 immunostaining in the outer part of cytoplasm along the cell membrane (Fig. 1B). CK7 expression was positive in 28 of the $126(22 \%)$ ESCCs, and negative in 98 (78\%). CK7-positive was significantly associated with poor differentiation of ESCCs (Table I).

CK14 expression and clinicopathological parameters of $E S C C$. CK14 expression was seen in the cytoplasm of the 

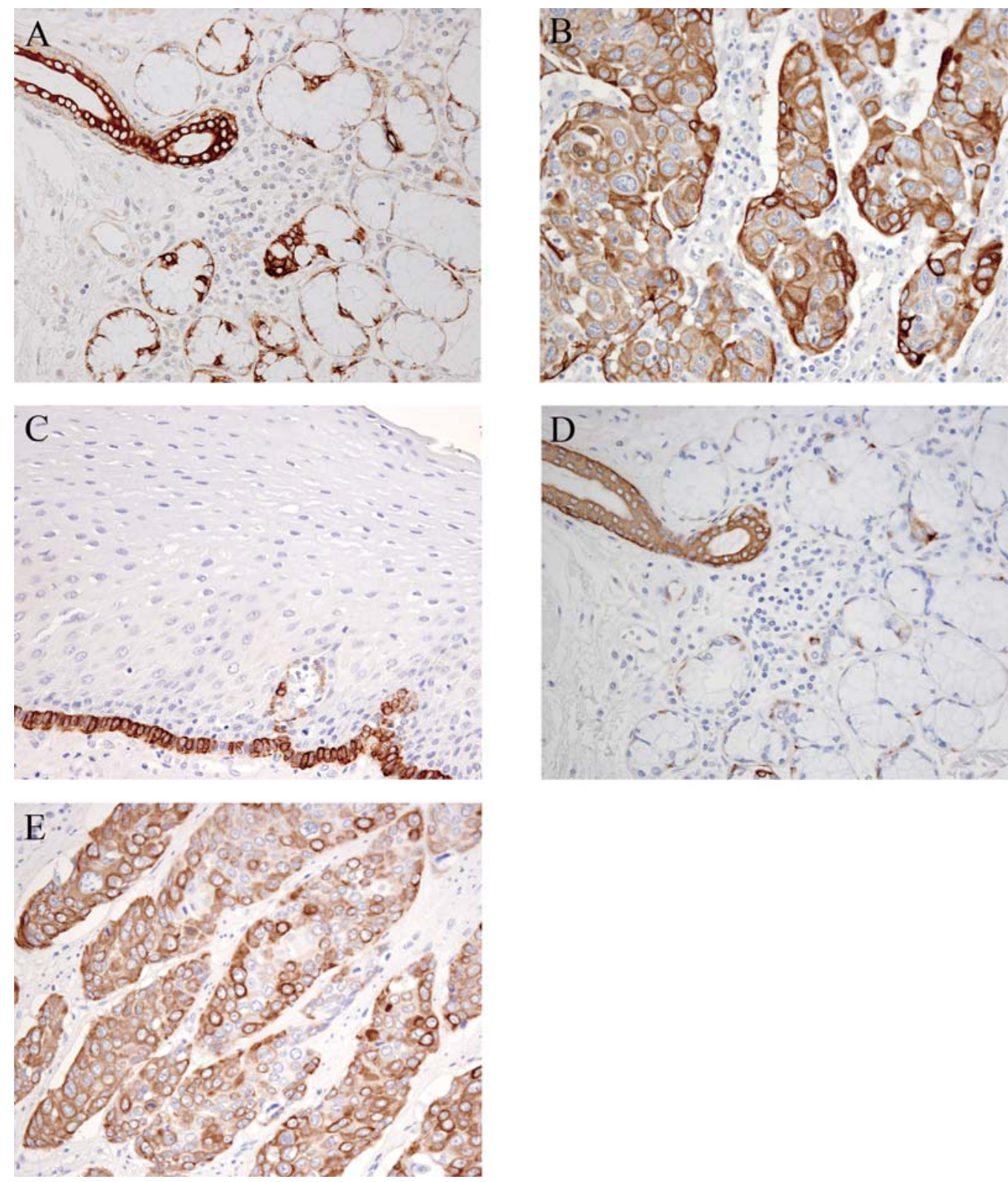

Figure 1. Immunohistochemical staining of non-neoplastic adjacent esophagus (A, C and D) and ESCC (B and E) (original magnification, x200). (A), CK7 expression was seen in the duct and the glandular epithelium of non-neoplastic esophageal glands. (B), CK7 expression was observed in the cytoplasm of ESCC cells. Strong CK7 immunostaining in the outer part of the cytoplasm along cell membrane can be seen. (C and D), CK14 expression was seen in the basal cells of non-neoplastic esophageal epithelium (C) and in the duct and the glandular epithelium of esophageal glands (D). (E), CK14 expression was observed in the cytoplasm of ESCC cells. Some ESCC cells showed strong immunoreactivity along the cell membrane, similar to CK7.

basal cells of adjacent non-neoplastic esophageal epithelium (Fig. 1C) as well as of the duct and glandular epithelium of the esophageal glands (Fig. 1D). CK14 expression was also observed in the cytoplasm of many cancer cells, and strong cytoplasmic staining along the cell membrane was occasionally present (Fig. 1E). CK14 expression was positive in 106 of $126(84 \%)$ of ESCCs, and negative in 20 (16\%). CK14-negative was significantly associated with poor differentiation, lymphatic invasion, and advanced stages of ESCCs (Table I).

CK7 and CK14 expression and the survival of the ESCC patients. Fig. 2 shows the Kaplan-Meier cumulative overall survival curves according to CK7 and CK14 expression.
CK7-positive and CK14-negative were significantly associated with worse survival rate.

Survival analyses according to $C K 7$ and $C K 14$ expression in stage I/IIA/IIB and stage III/IV patients. We performed survival analyses of the patients by dividing them into two groups according to TNM stage to assess the prognostic impact of CK7 and CK14 expression in the early stages and advanced stages of ESCC. CK7-positive was significantly associated with worse survival rate of patients with stage I/ IIA/IIB ESCCs (Fig. 3). The 5-year overall survival rate of stage I/IIA/IIB patients with CK7-positive and CK7-negative tumors was 50.0 and $90.3 \%$, respectively. There was no significant association between CK14 expression and the 
Table I. Associations between expression of CK7 and expression of CK14 and clinicopathological parameters of ESCC.

\begin{tabular}{|c|c|c|c|c|c|c|}
\hline & \multicolumn{2}{|c|}{ CK7 expression } & \multirow[b]{2}{*}{ P-value } & \multicolumn{2}{|c|}{ CK14 expression } & \multirow[b]{2}{*}{ P-value } \\
\hline & $\begin{array}{l}\text { Positive } \\
(\mathrm{n}=28)\end{array}$ & $\begin{array}{l}\text { Negative } \\
(n=98)\end{array}$ & & $\begin{array}{l}\text { Positive } \\
(n=106)\end{array}$ & $\begin{array}{l}\text { Negative } \\
(n=20)\end{array}$ & \\
\hline \multicolumn{7}{|l|}{ Age } \\
\hline$\geq \operatorname{Median}(\mathrm{n}=65)$ & 19 & 46 & 0.057 & 55 & 10 & $>0.999$ \\
\hline$<$ Median $(\mathrm{n}=61)$ & 9 & 52 & & 51 & 10 & \\
\hline \multicolumn{7}{|l|}{ Gender } \\
\hline Male (n=107) & 24 & 83 & $>0.999$ & 89 & 18 & 0.736 \\
\hline Female $(n=19)$ & 4 & 15 & & 17 & 2 & \\
\hline \multicolumn{7}{|l|}{ Tumor size } \\
\hline$\geq$ Median $(n=63)$ & 14 & 49 & $>0.999$ & 55 & 8 & 0.339 \\
\hline$<$ Median $(\mathrm{n}=62)$ & 14 & 48 & & 50 & 12 & \\
\hline \multicolumn{7}{|l|}{ Histologic grade } \\
\hline $\begin{array}{l}\text { Well/moderately } \\
\text { differentiated }(n=103)\end{array}$ & 19 & 84 & 0.049 & 93 & 10 & $<0.001$ \\
\hline Poorly differentiated $(n=23)$ & 9 & 14 & & 13 & 10 & \\
\hline \multicolumn{7}{|l|}{ Depth of invasion } \\
\hline $\mathrm{T} 1 / \mathrm{T} 2(\mathrm{n}=67)$ & 19 & 48 & 0.089 & 60 & 7 & 0.091 \\
\hline $\mathrm{T} 3 / 4(\mathrm{n}=59)$ & 9 & 50 & & 46 & 13 & \\
\hline \multicolumn{7}{|l|}{ Lymph node metastasis } \\
\hline Positive $(\mathrm{n}=78)$ & 16 & 62 & 0.660 & 63 & 15 & 0.219 \\
\hline Negative $(n=48)$ & 12 & 36 & & 43 & 5 & \\
\hline \multicolumn{7}{|l|}{ Distant metastasis } \\
\hline Positive $(n=5)$ & 1 & 4 & $>0.999$ & 5 & 0 & $>0.999$ \\
\hline Negative $(n=121)$ & 27 & 94 & & 101 & 20 & \\
\hline \multicolumn{7}{|l|}{ Lymphatic invasion } \\
\hline Positive $(n=64)$ & 17 & 47 & 0.286 & 49 & 15 & 0.027 \\
\hline Negative $(n=62)$ & 11 & 51 & & 57 & 5 & \\
\hline \multicolumn{7}{|l|}{ Venous invasion } \\
\hline Positive $(\mathrm{n}=76)$ & 13 & 63 & 0.125 & 64 & 12 & $>0.999$ \\
\hline Negative $(n=50)$ & 15 & 35 & & 42 & 8 & \\
\hline \multicolumn{7}{|l|}{ Stage } \\
\hline I/IIA/IIB (n=78) & 20 & 58 & 0.276 & 70 & 8 & 0.043 \\
\hline III/IV (n=48) & 8 & 40 & & 36 & 12 & \\
\hline
\end{tabular}

A

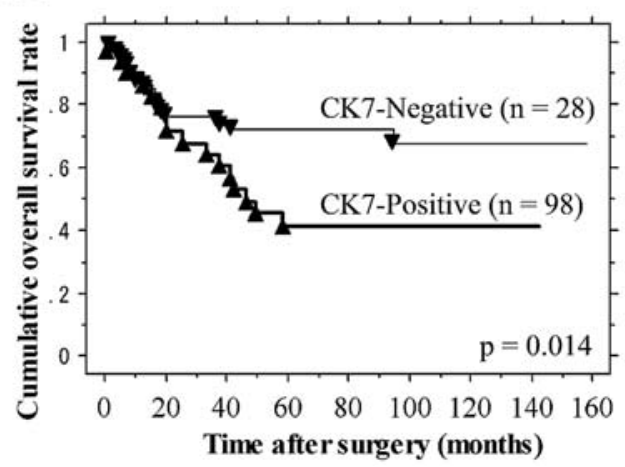

B

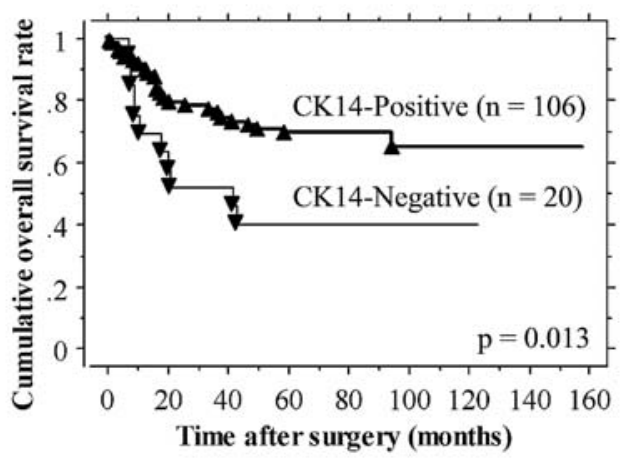

Figure 2. The Kaplan-Meier overall survival curves for ESCC patients according to CK7 (A) and CK14 (B) expression. 
A

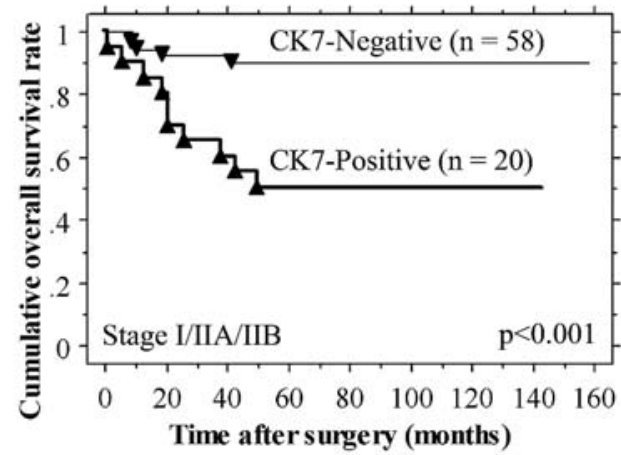

C

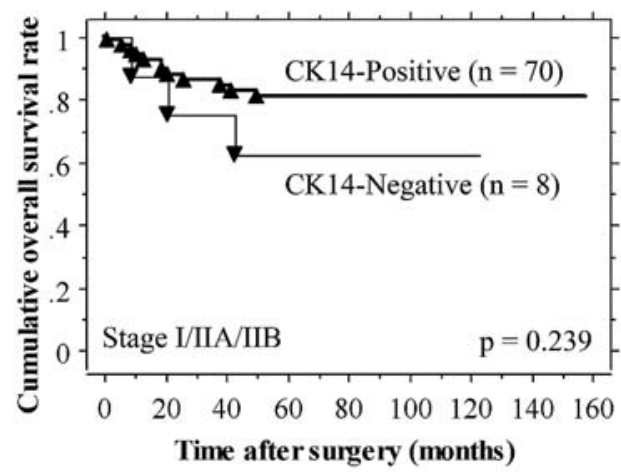

B

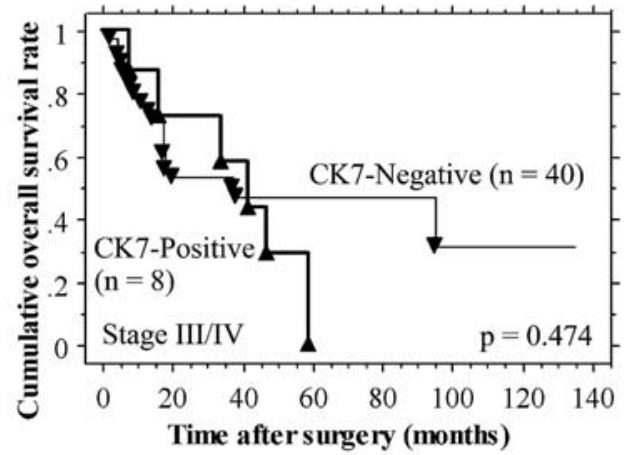

D

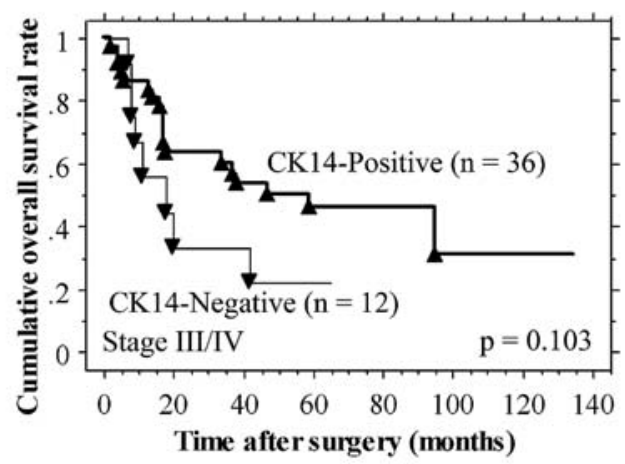

Figure 3. The Kaplan-Meier overall survival curves of the group with stage I/IIA/IIB ESCC (A and C) and the group with stage III/IV ESCC (B and D), according to CK7 (A and B) and CK14 (C and D) expression.

A

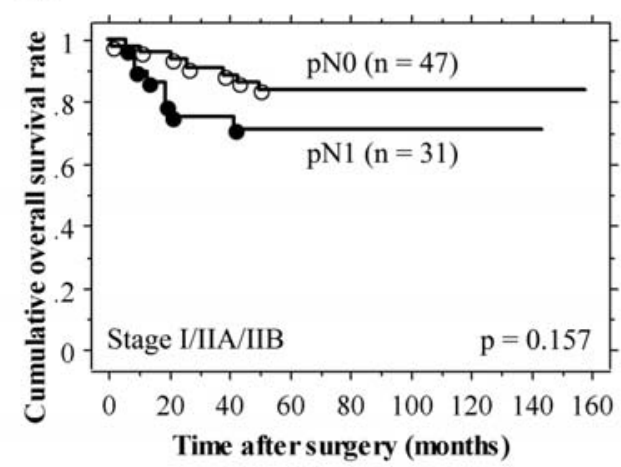

C

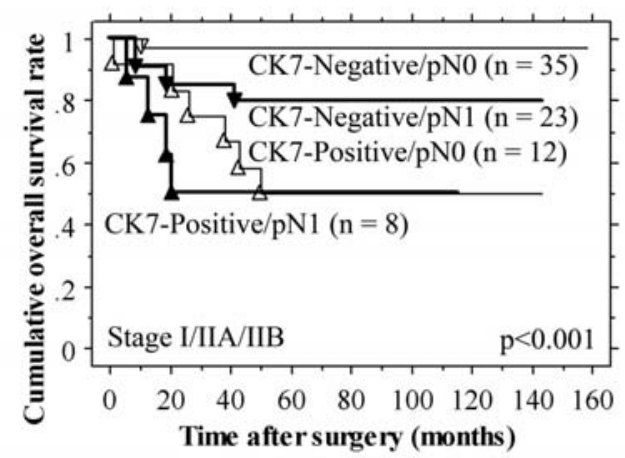

B

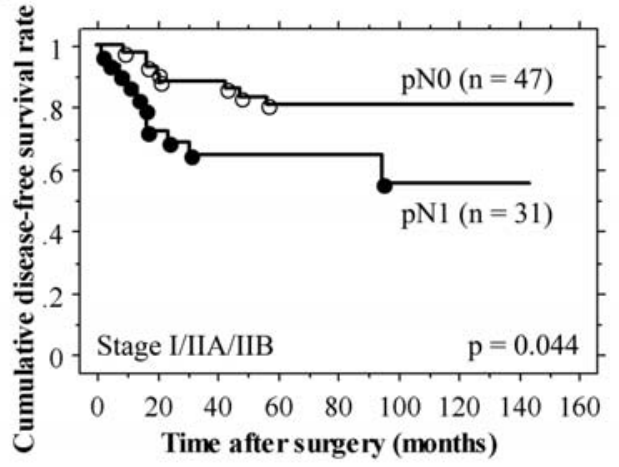

D

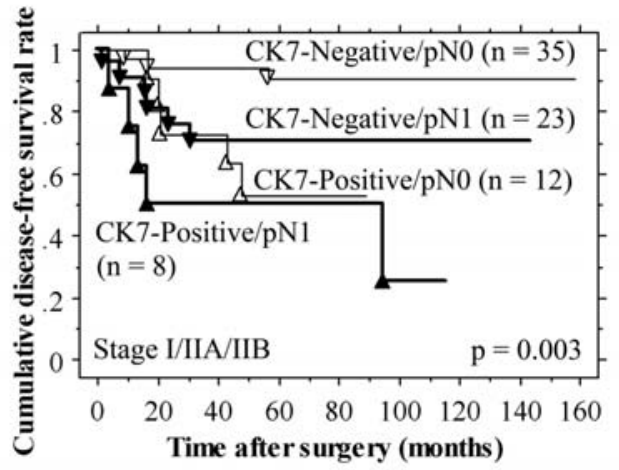

Figure 4. The Kaplan-Meier overall (A and C) and disease-free (B and D) survival curves for stage I/IIA/IIB patients according to nodal status (A and B) and a combination of CK7 expression and nodal status (C and D). 
Table II. Univariate and multivariate survival analyses of the stage I/IIA/IIB ESCC patients by the Cox proportional hazards model.

\begin{tabular}{|c|c|c|c|c|c|c|}
\hline \multirow[b]{2}{*}{ Variables } & \multicolumn{3}{|c|}{ Univariate analysis } & \multicolumn{3}{|c|}{ Multivariate analysis } \\
\hline & $\begin{array}{l}\text { Hazard } \\
\text { ratio }\end{array}$ & $\begin{array}{c}95 \% \text { confidence } \\
\text { interval }\end{array}$ & P-value & $\begin{array}{l}\text { Hazard } \\
\text { ratio }\end{array}$ & $\begin{array}{c}95 \% \text { confidence } \\
\text { interval }\end{array}$ & P-value \\
\hline \multicolumn{7}{|l|}{ Age } \\
\hline$\geq$ Median vs $<$ median & 2.82 & $0.90-8.87$ & 0.076 & 2.28 & $0.69-7.56$ & 0.179 \\
\hline \multicolumn{7}{|l|}{ Tumor location } \\
\hline Upper vs lower & 2.38 & $0.63-9.02$ & 0.201 & & & \\
\hline Middle vs lower & 0.51 & $0.15-1.70$ & 0.273 & & & \\
\hline \multicolumn{7}{|l|}{ Tumor size } \\
\hline$\geq$ Median vs $<$ median & 0.78 & $0.27-2.29$ & 0.654 & & & \\
\hline \multicolumn{7}{|l|}{ Histologic grade } \\
\hline Poorly vs well/moderate & 2.51 & $0.86-7.33$ & 0.094 & 1.05 & $0.32-3.46$ & 0.934 \\
\hline \multicolumn{7}{|l|}{ Depth of invasion } \\
\hline T3/T4 vs Tis/T1/T2 & 0.33 & $0.04-2.54$ & 0.290 & & & \\
\hline \multicolumn{7}{|l|}{ Lymph node metastasis } \\
\hline Present vs absent & 2.05 & $0.74-5.65$ & 0.167 & & & \\
\hline \multicolumn{7}{|l|}{ Lymphatic invasion } \\
\hline Present vs absent & 4.49 & $1.43-14.14$ & 0.010 & 3.81 & $1.17-12.44$ & 0.027 \\
\hline \multicolumn{7}{|l|}{ Venous invasion } \\
\hline Present vs absent & 1.01 & $0.36-2.77$ & 0.993 & & & \\
\hline \multicolumn{7}{|l|}{ CK7 expression } \\
\hline Positive vs negative & 6.16 & $2.10-18.04$ & $<0.001$ & 3.83 & $1.12-13.11$ & 0.032 \\
\hline \multicolumn{7}{|l|}{ CK14 expression } \\
\hline Positive vs negative & 0.48 & $0.14-1.69$ & 0.252 & & & \\
\hline
\end{tabular}

survival of stage I/IIA/IIB ESCC patients. Expression of neither protein was significantly associated with the survival of the stage III/IV patients.

Because lymph node metastasis is reported to be an independent prognostic factor of early esophageal cancer, including early ESCC (17), we examined the impact of CK7 expression on the survival of stage I/IIA/IIB patients in relation to nodal status. The Kaplan-Meier curves showed that the stage I/IIA/IIB ESCC patients without lymph node metastasis (pN0) had a significantly better disease-free survival rate than the patients with lymph node metastasis (pN1), although the difference in overall survival rate between the groups was not significant (Fig. 4A and B). When CK7 expression was combined with nodal status, the group with CK7-positive tumors had a worse overall survival rate and disease-free survival rate in both the $\mathrm{pN} 0$ and $\mathrm{pN} 1$ subgroups (Fig. 4C and D). It is noteworthy that the CK7-positive/pN0 group had a worse survival rate than the CK7-negative/pN1 group. The 5-year overall survival rate of the CK7-positive/pN1 group, CK7-positive/pN0 group, CK7-negative/pN1 group and CK7-negative/pN0 group was 50.0, 50.0, 79.5 and 97.0\%, respectively.

We performed analyses using the Cox proportional hazards model to identify factors involved in survival of stage I/IIA/IIB ESCC patients. The univariate analysis showed that lymphatic invasion and CK7-positivity were significant prognostic factors, and they were identified as independent prognostic factors for patients with stage I/IIA/IIB ESCC in the multivariate analysis (Table II).

\section{Discussion}

The result of this study shows that expression of CK7 and expression of CK14 assessed immunohistochemically were significantly associated with the survival of ESCC patients, and CK7 expression was found to be a significant predictor of poor clinical outcome and an independent prognostic factor for patients with stage I/IIA/IIB ESCC. Surprisingly, CK7 expression had a great impact on the outcome of stage I/ IIA/IIB patients, and it was even greater than the impact of nodal status. We set the cut-off level for both CK7 and CK14 at $10 \%$ as in the study on gastric carcinoma by Kim et al (9). We also tested multiple cut-off levels, and the results confirmed that the $10 \%$ cut-off value for both proteins was most discriminative for patients' outcome in our series (data not shown).

Ando et al reported that postoperative adjuvant chemotherapy significantly improved the disease-free survival rate 
of stage IIA-IV ESCC patients in a multicenter randomized controlled trial (4). They also found marked risk reduction by postoperative chemotherapy in the subgroup with lymph node metastasis and/or higher pT stage, but not in the subgroup without lymph node metastasis. Their results may be attributable to a lack of appropriate stratification of early TNM stage ESCCs. If it were possible to predict the outcome of early TNM stage ESCC patients, those with a poor outcome would be candidates for more intensive multimodal treatment instead of standard surgery alone. The benefit of multimodal treatment might become more significant if the subject group were more clearly defined. Since CK7 expression clearly stratified the survival of early stage patients, it may be a useful biomarker for selecting patients for multimodal treatment. However, a prospective study should be carried out to test the hypothesis that stratification of patients according to CK7 expression contributes to choosing optimal treatment in individual cases, and in turn improves the survival of early stage ESCC patients.

Although we showed an association between expression of CK7 and CK14 and the outcome of ESCC patients, the mechanisms underlying the associations remain unknown. In normal tissue, distinct subsets of CKs are expressed based on the epithelial cell type and the degree of differentiation. In non-neoplastic esophagus, CK7 expression was present in the duct and the glandular epithelium of esophageal glands, but not in squamous epithelium. On the other hand, CK14 was positive in both the basal cells of stratified squamous epithelium and in the duct and the glandular epithelium of esophageal glands. Thus, the patterns of expression of these two CKs seem to reflect the cellular phenotype. The same as non-neoplastic esophageal squamous epithelium, the majority of the ESCCs were negative for CK7 and positive for CK14. Interestingly, patients with ESCCs that were CK7positive and/or CK14-negative, in contrast to their nonneoplastic counterpart, had a poor clinical outcome. Expression of these two CKs may reflect distinct phenotypes of ESCC cells that possess distinct patterns of expression of multiple genes, and as a result exhibit different biological behavior (Sano et al, unpublished data). Further study is necessary to determine whether this is true.

In conclusion, ESCCs that were positive for CK7 expression and negative for CK14 expression were associated with worse survival rate. CK7 expression was an independent prognostic factor for stage I/IIA/IIB ESCCs, and enabled stratification of the clinical outcome of this group of patients. Thus, CK7 expression may be a useful biomarker for choosing the optimal treatment for individual patients with stage I/IIA/ IIB ESCC.

\section{Acknowledgements}

This study was supported in part by the program for promotion of Fundamental Studies in Health Sciences of the National Institute of Biomedical Innovation, in part by a Grant-in-Aid for the Third Comprehensive 10-Year Strategy for Cancer Control and for Cancer Research (16-15) from the Ministry of Health, Labour and Welfare of Japan. A.Y., M.S. and H.D. were awardees of Research Resident Fellowships from the Foundation for Promotion of Cancer Research (Japan) for the 3rd Term Comprehensive 10-Year Strategy for Cancer Control.

\section{References}

1. Ando N, Ozawa S, Kitagawa Y, Shinozawa Y and Kitajima M: Improvement in the results of surgical treatment of advanced squamous esophageal carcinoma during 15 consecutive years. Ann Surg 232: 225-232, 2000.

2. Enzinger PC and Mayer RJ: Esophageal cancer. N Engl J Med 349: 2241-2252, 2003.

3. Veuillez V, Rougier P and Seitz JF. Multimodal treatment of oesophageal cancer. Best Pract Res Clin Gastroenterol 21: 947-963, 2007.

4. Ando N, Iizuka T, Ide H, et al: Surgery plus chemotherapy compared with surgery alone for localized squamous cell carcinoma of the thoracic esophagus: a Japan clinical oncology group study-JCOG9204. J Clin Oncol 21: 4592-4596, 2003.

5. Gebski V, Burmeister B, Smithers BM, Foo K, Zalcberg J and Simes J: Survival benefits from neoadjuvant chemoradiotherapy or chemotherapy in oesophageal carcinoma; a meta-analysis. Lancet Oncol 8: 226-234, 2007.

6. Nozoe T, Saeki H, Ohta T and Sugimachi K: Clinicopathological features of early esophageal squamous cell carcinoma with subsequent recurrence. Dis Esophagus 15: 145-148, 2002.

7. Schweizer J, Bowden PE, Coulombe PA, et al: New consensus nomenclature for mammalian keratins. J Cell Biol 174: 169-174, 2006.

8. Chu PG and Weiss LM: Keratin expression in human tissues and neoplasms. Histopathology 40: 403-439, 2002.

9. Kim MA, Lee HS, Yang HK and Kim WH: Cytokeratin expression profile in gastric carcinomas. Hum Pathol 35: 576-581, 2004.

10. Lam KY, Loke SL, Shen XC and Ma LT: Cytokeratin expression in non-neoplastic oesophageal epithelium and squamous cell carcinoma of the oesophagus. Virchows Arch 426: 345-349, 1995.

11. Takahashi H, Shikata N, Senzaki H, Shintaku M and Tsubura A: Immunohistochemical staining patterns of keratins in normal oesophageal epithelium and carcinoma of the oesophagus. Histopathology 26: 45-50, 1995.

12. Xue LY, Hu N, Song YM, et al: Tissue microarray analysis reveals a tight correlation between protein expression pattern and progression of esophageal squamous cell carcinoma. BMC Cancer 6: 296, 2006.

13. Gharib TG, Chen G, Wang H, et al: Proteomic analysis of cytokeratin isoforms uncovers association with survival in lung adenocarcinoma. Neoplasia 4: 440-448, 2002

14. Uenishi T, Kubo S, Yamamoto T, et al: Cytokeratin 19 expression in hepatocellular carcinoma predicts early postoperative recurrence. Cancer Sci 94: 851-857, 2003.

15. Sobin LH and Wittekind C (eds): TNM classification of malignant tumors. 6th edition. Wiley-Liss, New York, pp60-64, 2002.

16. Hamilton SR and Aaltonen LA (eds): World Health Organization classification of tumors: pathology and genetics of tumors of the digestive system. IARC Press, Lyon, pp11-19, 2000.

17. Stein HJ, Feith M, Bruecher BLDM, Naehrig J, Sarbia M and Siewert JR: Early esophageal cancer. Pattern of lymphatic spread and prognostic factors for long-term survival after surgical resection. Ann Surg 242: 566-575, 2005. 